\title{
Lateral medullary syndrome: a diagnostic approach illustrated through case presentation and literature review
}

\author{
Gregory S. Day, MD, MSc ${ }^{*}$; Richard H. Swartz, MD, PhD**; Jordan Chenkin, MD, MEd ${ }^{\ddagger \S}$; \\ Adil I. Shamji, MD"; David W. Frost, $\mathrm{MD}^{+\uparrow \#}$
}

\section{ABSTRACT}

Patients with lateral medullary syndrome classically present with crossed hemisensory disturbance, ipsilateral Horner syndrome, and cerebellar signs, all of which are attributable to infarction of the lateral medulla. However, variability in the presentation of this syndrome is the rule, as illustrated in this case presentation and literature review. We propose an approach to diagnosis and management of the lateral medullary syndrome and illustrate the need to integrate clinical information with an understanding of brainstem anatomy with the goal of determining which patients require urgent neuroimaging and acute stroke therapies. The importance of recognition of this condition in the emergency department is underscored by the association between lateral medullary infarction and vertebral artery dissection. With optimal therapy, the prognosis for recovery from lateral medullary syndrome is good.

\section{RÉSUMÉ}

Les patients qui souffrent du syndrome bulbaire latéral présentent généralement des troubles hémisensoriels croisés, un syndrome de Claude Bernard-Horner homolatéral et des signes cérébelleux, manifestations qui résultent toutes d'un infarctus du bulbe latéral. Toutefois, le tableau clinique habituel du syndrome est variable, comme en témoignent un exposé de cas et l'examen de la documentation. Nous ferons donc état de l'approche diagnostique et de la prise en charge du syndrome bulbaire latéral, et soulignerons la nécessité de rassembler tous les renseignements d'ordre clinique et de les mettre en relation avec l'anatomie du tronc cérébral afin de distinguer les patients devant subir d'urgence des examens en neuro-imagerie et des traitements pour un accident vasculaire cérébral. L'association entre l'infarctus du bulbe latéral et la dissection de l'artère vertébrale fait ressortir l'importance de reconnaître cette première affection au service des urgences. Moyennant le meilleur traitement possible, le syndrome bulbaire latéral porte un pronostic favorable quant au rétablissement.

Keywords: lateral medullary infarct, lateral medullary syndrome, posterior fossa, stroke, vertebral artery dissection, Wallenberg syndrome

The lateral medullary (Wallenberg) syndrome arises from compromise of the posterior inferior cerebellar artery (PICA) leading to infarction of the lateral medulla. Patients with the complete syndrome present with crossed hemisensory disturbance (ipsilateral face, contralateral body), ipsilateral Horner syndrome, and ipsilateral cerebellar signs. A historical article published in 1961 estimated that the syndrome accounts for $2.5 \%$ of ischemic strokes ${ }^{1}$; however, given the diagnostic challenges involved, this is likely an underestimate. Accurate interpretation of clinical signs and symptoms is critical to establishing the diagnosis and determining which patients require urgent neuroimaging and acute stroke therapies. Clinical recognition of patients with lateral medullary infarction is of particular importance due to its association with vertebral artery dissection in 15 to $26 \%$ of cases $^{2,3}$ and the favourable prognosis associated with optimal management.

\footnotetext{
From the *Division of Neurology, Department of Medicine, University of Toronto; †University Health Network Hospitals; $¥$ Sunnybrook Health Sciences Centre; and §Division of Emergency Medicine, Department of Medicine, University of Toronto; ॥Department of Family and Community Medicine, University of Toronto; $\uparrow$ Division of General Internal Medicine, Department of Medicine, University of Toronto and \#Herbert HoPingKong Centre for Excellence in Education and Practice, University of Toronto, Toronto, ON.
}

Correspondence to: Dr. Gregory Day, Division of Neurology, University of Toronto, Toronto Western Hospital, 399 Bathurst Street, Toronto, ON M5T 2S8; gregg.day@mail.utoronto.ca.

This article has been peer reviewed. 


\section{CASE PRESENTATION}

A previously well 67-year-old man presented to the emergency department (ED) with a 4-hour history of vertigo, nausea, and vomiting. He had a 50-pack-year smoking history and was not taking any medications. There was no history of neck trauma or manipulation. While in the ED, his condition deteriorated. He developed slurred speech, numbness and paresthesias of the left hemibody (with preserved facial sensation), and difficulty swallowing liquids.

His blood pressure was 195/90 $\mathrm{mm} \mathrm{Hg}$, and his pulse was regular at 66 beats $/ \mathrm{min}$. Cardiovascular, respiratory, and abdominal examinations were all unremarkable, and his mental status was normal. Verbal comprehension, naming, and repetition were normal; however, his speech was dysarthric. Cranial nerve examination revealed right-sided ptosis and miosis, consistent with a partial Horner syndrome. The patient's visual fields were normal on confrontational testing, and his extraocular movements were full and without nystagmus. There was symmetrical contraction of the muscles of facial expression. Pinprick sensation was decreased throughout the right face, and his right corneal reflex was absent. His uvula was deviated to the left, and his gag reflex was absent. There was mild pyramidal pattern weakness in his right arm and leg (flexors weaker than extensors in the arm; extensors weaker than flexors in the leg). Deep tendon and superficial reflexes were normal. Sensation to pain and temperature was decreased below the neck on the left side. Coordination assessment with finger-to-nose and heel-to-shin testing revealed severe right-sided dysmetria. When sitting or standing, the patient had marked truncal ataxia, with a tendency to fall to the right. He was unable to ambulate.

Routine laboratory investigations and an electrocardiogram were normal. An unenhanced (noncontrast) computed tomographic (CT) scan of the brain was normal. The diagnosis of lateral medullary infarction was made on clinical grounds. Acute treatment with intravenous tissue plasminogen activator was not offered due to the delayed presentation (clinical assessment was completed 6 hours following symptom onset). Magnetic resonance angiography (MRA) of the head and neck vessels showed occlusion of the proximal right vertebral artery, suspicious for extracranial dissection (Figure 1A). The PICA was patent (Figure 1B). with supply through collateral flow via the basilar and left vertebral arteries. Magnetic resonance imaging (MRI)
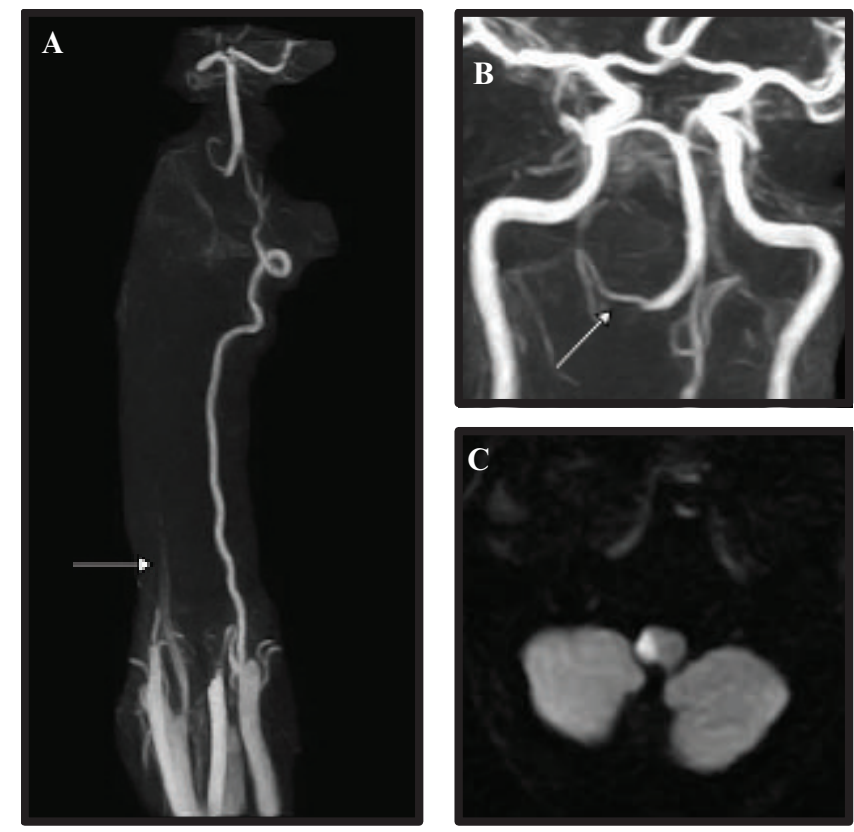

Figure 1. $A$, Magnetic resonance angiogram of vertebral arteries confirming occlusion of the proximal right vertebral artery (arrow). B, The posterior inferior cerebellar artery is visualized (arrow). C, Diffusion-weighted image showing an area of restricted diffusion in the right lateral medulla, compatible with acute ischemic infarct.

of the brain identified an area of restricted diffusion in the right lateral medulla (Figure 1C).

Within 48 hours, he improved dramatically. His speech returned to normal, his dysmetria resolved, and he was able to sit unassisted. Fasting blood glucose was normal, and no arrhythmias were identified with 48 hours of cardiac monitoring. The patient's dysphagia persisted, requiring placement of a gastric tube. Blood pressure control was optimized with dual antihypertensive therapy (an angiotensin-converting enzyme inhibitor and a thiazide diuretic). A statin was commenced for management of newly diagnosed dyslipidemia. Smoking cessation counselling was provided, and warfarin was commenced for ongoing anticoagulation. Ten days after admission, the patient was eating modified-consistency meals and was transferred to a stroke rehabilitation centre. At 3 months postdischarge, he had recovered almost entirely (Modified Rankin Scale $[\mathrm{MRS}]=1^{4}$ ). He no longer required the gastric tube and was ambulating independently. Repeat neurovascular imaging prior to the 6month follow-up showed recanalization of the vertebral artery. His warfarin was discontinued, and antiplatelet therapy (acetylsalicylic acid) was prescribed for longterm secondary stroke prevention. 


\section{DISCUSSION}

The triad of Horner syndrome, ipsilateral ataxia, and contralateral hypoalgesia clinically identifies the patient with lateral medullary syndrome ${ }^{5,6}$; however, the diagnosis should be considered in all patients with sudden-onset symptoms and signs localizing to the medulla. Table 1 shows the pooled sensitivity of various symptoms and signs compiled from the largest case series in the literature (specificity has not been determined through prospective observation but is likely low). ${ }^{3,7,8}$ Crossed hemisensory deficits are reported in $90 \%$ of reviewed cases and thus should be regarded as a highly sensitive finding., ${ }^{3,7} 8$ Vertebral artery dissection and large artery atherosclerosis are important risk factors for developing lateral medullary syndrome, accounting for the majority of cases reported in stroke registries., ${ }^{2,9}$ Vertebral artery dissection is most common in younger patients or those with a history of trauma, whereas atherosclerosis is more likely in older patients with a history of hypertension, diabetes, smoking, and coronary artery disease., ${ }^{2,10,11}$ Embolic stroke originating from the heart is another potential etiology that should be considered, particularly in patients with arrhythmias, cardiac dysfunction, or valvular disease. ${ }^{12}$

\section{Anatomy}

The classic lateral medullary syndrome results from damage to the trigeminal spinal nucleus and tract, spinothalamic tract, descending sympathetic fibres, inferior cerebellar peduncle, vestibular nuclei, and nucleus ambiguus (Figure 2). Variations arise when areas at risk are preserved through residual perfusion or collateral flow or when perfusion in surrounding areas is compromised. Patients with infarction predominantly affecting the caudal medulla tend to present with vertigo, nystagmus, and ataxia owing to involvement of vestibular nuclei and cerebellar outflow tracts. ${ }^{13}$ More rostral lesions involving the nucleus ambiguus (the motor nucleus of the glossopharyngeal and vagus nerves) may occur and present with severe dysphagia and hoarseness. Rarely, these may be the only presenting complaints. Patients may complain of loss of taste owing to involvement of the nucleus solitarius. Occasionally, involvement of the caudal pons produces ipsilateral facial paresis through involvement of the facial nucleus. The corticospinal tracts (motor function), hypoglossal nuclei (tongue movement), dorsal column medial lemniscus pathways, and associated nucleus gracilis and cuneatus (vibration and position sense below the neck) are all supplied by the anterior spinal artery and are therefore usually spared.

\section{Diagnosis and management}

The approach to the patient with suspected lateral medullary syndrome requires rapid assessment, a clear determination of time of symptom onset, and the performance of a neurologic examination focused on discriminating infarction from mimic (Figure 3). ${ }^{14,15} \mathrm{In}$ a prospective cross-sectional study of 101 patients

Table 1. Pooled sensitivity of symptoms and signs in radiographically proven lateral medullary infarct from the largest case series $^{3,7,8}$

\begin{tabular}{lll}
\hline Symptom/sign & \multicolumn{1}{c}{ Nuclei/tracts affected } & Pooled sensitivity \\
\hline $\begin{array}{l}\text { Crossed-sensory deficit (ipsilateral } \\
\text { face, contralateral body) }\end{array}$ & Trigeminal nucleus and tract; spinothalamic tract & $0.90^{*}$ \\
Vertigo & Inferior cerebellar peduncle; vestibular nuclei & $0.81^{*}$ \\
Cerebellar ataxia (ipsilateral) & Inferior cerebellar peduncle & $0.77^{*}$ \\
Horner syndrome (ipsilateral) & Descending sympathetic tract & $0.76^{*}$ \\
Dysphagia & Nucleus ambiguus & $0.60^{* *}$ \\
Nystagmus & Vestibular nuclei & $0.57^{* *}$ \\
Nausea and/or vomiting & Vestibular nuclei & $0.55^{* *}$ \\
Dysarthria & Nucleus ambiguus & $0.52^{*}$ \\
Headache & Vertebral artery dissection & $0.48^{*}$ \\
Diminished gag reflex (ipsilateral) & Nucleus ambiguus & $0.64^{* * *}$ \\
Hoarseness & Nucleus ambiguus & $0.63^{* * *}$ \\
Skew deviation of eyes & Vestibular nuclei & $0.41^{* * *}$ \\
\hline${ }^{*} n=326 ;{ }^{* *} n=296 ;{ }^{* *} n=29$. & &
\end{tabular}



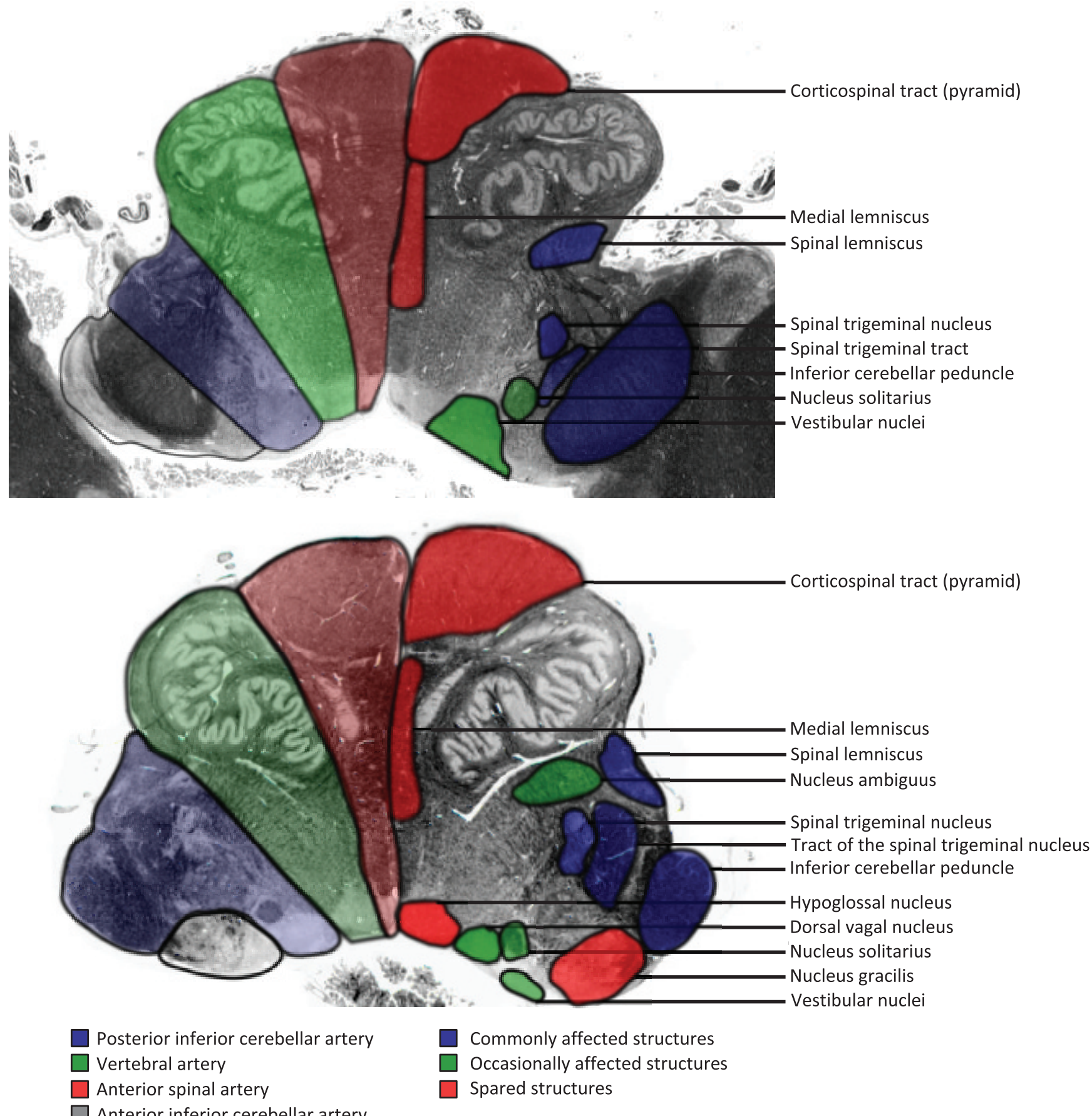

Figure 2. Anatomy of the medulla oblongata. Vascular supply (left) and relevant anatomic structures (right) are shown superimposed on axial slices of the rostral (top) and caudal (bottom) medulla. Commonly affected structures (b/ue) refer to structures involved in $>75 \%$ of cases reported in the largest case series. ${ }^{3,7,8}$ Occasionally, involved structures (green) refer to structures involved in 50 to $75 \%$ of cases. Adapted with permission from Stewart $P$ et al. ${ }^{24}$

presenting with "acute vestibular syndrome," the addition of a simple three-step oculomotor examination identified patients with stroke with $100 \%$ sensitivity and $96 \%$ specificity and clinically identified all 17 patients with lateral medullary syndrome. ${ }^{15}$
These bedside manoeuvres are summarized by the mnemonic HINTS (Head-Impulse-Nystagmus-Testof-Skew) and should be performed in all patients presenting with subtle symptoms suggestive of posterior fossa infarction, including isolated vertigo, nausea and 


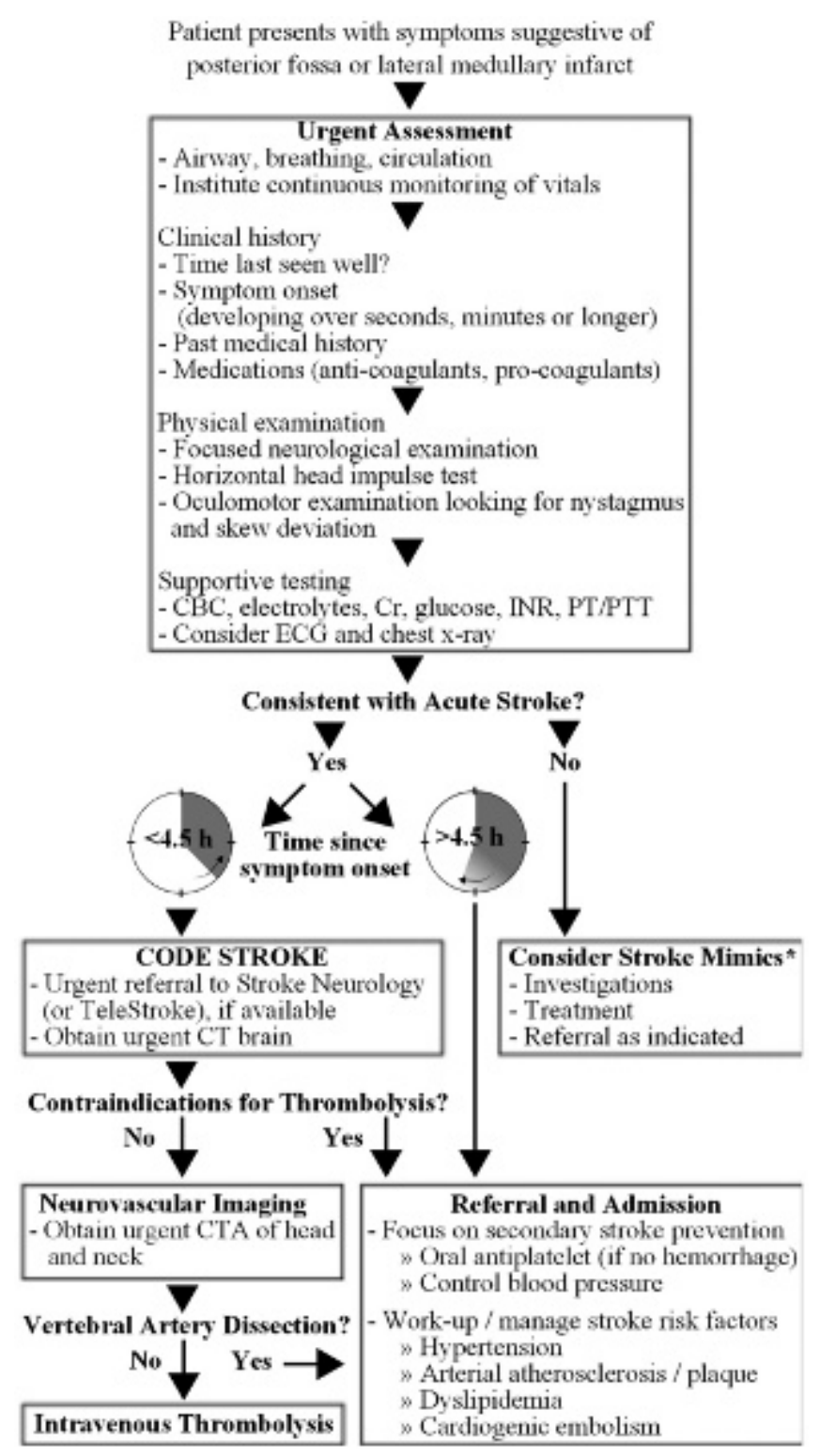

Figure 3. Suggested approach for the emergency department (ED) patient presenting with sudden-onset symptoms suggestive of acute posterior fossa or lateral medullary infarct. This approach is consistent with Canadian Best Practice Recommendations for Stroke Care, which emphasizes urgent assessment, with the goal of selecting patients likely to benefit from acute thrombolytic therapy within 60 minutes of ED presentation. ${ }^{20}{ }^{*}$ For review, see Tarnutzer and colleagues. ${ }^{14} \mathrm{CBC}=$ complete blood count; $\mathrm{CT}=$ computed tomography; CTA = computed tomographic angiography; ECG = electrocardiogram; INR = international normalized ratio; $\mathrm{PT}=$ prothrombin time; $\mathrm{PTT}=$ partial thromboplastin time.

vomiting, and gait intolerance. A central cause should be presumed, and investigations and treatment for acute stroke should be considered, in patients with any of the following: 1) a normal horizontal head impulse test (gaze is maintained with passive horizontal head thrust);
2) direction-changing nystagmus on eccentric gaze or vertical or torsional nystagmus; or 3) skew deviation (vertical ocular misalignment demonstrated with coveruncover testing of each eye). ${ }^{15}$

All patients with suspected acute stroke should receive urgent neuroimaging to exclude alternative diagnoses and to screen for contraindications to stroke therapies such as intracerebral hemorrhage, focal compression, or herniation. ${ }^{16}$ Unenhanced CT is well suited for this purpose and can be used with clinical assessment to select patients appropriate for treatment with intravenous thrombolytics. ${ }^{16}$ When indicated, additional neurovascular imaging should be performed screening for vertebral artery dissection. In most centres, computed tomographic angiography (CTA) is the modality of choice owing to widespread availability, speed of image acquisition, and minimal contraindications in patients without renal impairment. ${ }^{17}$ It is important to note that neither CT nor CTA is highly sensitive for the diagnosis of acute posterior fossa ischemic stroke; one study found that only $33 \%$ of acute MRI-confirmed brainstem infarctions were detected by CT. ${ }^{18}$ MRI (diffusion-weighted sequences \pm MRA) remains the gold standard test for the diagnosis of acute stroke, with an overall sensitivity of $83 \%$ and a specificity $96 \% .{ }^{19}$ Unfortunately, however, in the setting of acute lateral medullary infarction, even MRI may be unreliable. In the subset of patients with brainstem infarction, the sensitivity of MRI within 48 hours of symptom onset falls to $72 \%,{ }^{15}$ emphasizing the importance of clinical acumen when evaluating this patient population.

Canadian Best Practice Recommendations for Stroke Care stress that the goal of ED management is rapid assessment of patients with suspected acute stroke, with the goal of identifying patients likely to benefit from treatment with intravenous tissue plasminogen activator within 60 minutes of presentation. ${ }^{20}$ Current recommendations are that all patients presenting within 4.5 hours of symptom onset should be considered for treatment with intravenous tissue plasminogen activator, in consultation with specialty services managing acute stroke care (and via direct consultation or TeleStroke networks where available). ${ }^{20}$ An exception to this are patients with radiologically confirmed vertebral artery dissection with intradural extension, where best management is less clear due to the increased risk of subarachnoid hemorrhage. Although a retrospective review of outcomes following 
intravenous thrombolysis reported no difference in the rate of intracranial bleeding or recurrent ischemic strokes in patients with extradural arterial dissection, ${ }^{21}$ prospective trials are required before this treatment approach can be universally endorsed. Until further data are available, management decisions in such cases should be made on an individual basis, taking into account the clinical presentation, the degree of vascular compromise, and the availability of alternative endovascular therapies. In patients ineligible for treatment with thrombolytics, it remains equally important to identify those with infarction as such patients should be admitted to hospital for investigation and treatment focused on secondary stroke prevention..$^{20,22}$

With appropriate therapies, clinical monitoring, and post-stroke care, the prognosis for recovery from lateral medullary infarction remains favourable. The majority of patients have minimal deficits at 6 months, and over $85 \%$ achieve functional independence with ambulation $(\mathrm{MRS} \leq 3)$ within 1 year..$^{8,23}$

\section{CONCLUSIONS}

Recognition of the signs and symptoms associated with lateral medullary syndrome is important to patient care. Affected individuals should receive urgent neuroimaging to exclude alternate diagnoses and contraindications for acute stroke therapies. Whenever feasible, neurovascular imaging should be obtained to exclude vascular pathology. The favourable prognosis associated with lateral medullary syndrome distinguishes it from other posterior circulation strokes.

Acknowledgements: We thank our patient for his cooperation and willingness to participate in physician education. We also thank Drs. Anne Agur and Patricia Stewart for providing access to Functional Neuroanatomy software and advice concerning neuropathology figures and Dr. Cheryl Jaigobin for constructive comments and advice concerning the manuscript. We gratefully acknowledge the financial support of the Herbert HoPingKong Centre for Excellence in Education and Practice.

Competing interests: None declared.

\section{REFERENCES}

1. Currier RD, Giles CL, DeJong RN. Some comments on Wallenberg's lateral medullary syndrome. Neurology 1961; 11:778-91, doi:10.1212/WNL.11.9.778.

2. Lee MJ, Park YG, Kim SJ, et al. Characteristics of stroke mechanisms in patients with medullary infarction. Eur 7 Neurol 2012;19:1433-9, doi:10.1111/j.1468-1331.2012.03722.x.
3. Kim JS. Pure lateral medullary infarction: clinical-radiological correlation of 130 acute, consecutive patients. Brain 2003;126(Pt 8):1864-72, doi:10.1093/brain/awg169.

4. van Swieten JC, Koudstaal PJ, Visser MC, et al. Interobserver agreement for the assessment of handicap in stroke patients. Stroke 1988;19:604-7, doi:10.1161/01.STR.19.5.604.

5. Sacco RL, Freddo L, Bello JA, et al. Wallenberg's lateral medullary syndrome. Clinical-magnetic resonance imaging correlations. Arch Neurol 1993;50:609-14, doi:10.1001/archneur.1993.00540060049016.

6. Baugh CW, Brown DF, Nadel ES. Horner's syndrome, hoarseness, and unsteady gait. 7 Emerg Med 2009;36:176-80, doi:10.1016/j.jemermed.2008.12.015.

7. Kameda W, Kawanami T, Kurita K, et al. Lateral and medial medullary infarction: a comparative analysis of 214 patients. Stroke 2004;35:694-9, doi:10.1161/01.STR.0000117570.41153.35.

8. Fukuoka T, Takeda H, Dembo T, et al. Clinical review of 37 patients with medullary infarction. F Stroke Cerebrovasc Dis 2012; 21:594-9, doi:10.1016/j.jstrokecerebrovasdis.2011.01.008.

9. Caplan L. Posterior circulation ischemia: then, now and tomorrow. Stroke 2000;31:2011-23, doi:10.1161/01.STR. 31.8.2011.

10. Kratz SN, Butler KH. Vertebral artery dissection presenting as acute cerebrovascular accident. 7 Emerg Med 2011;40:1517, doi:10.1016/j.jemermed.2007.11.039.

11. Ernst E. Life-threatening complications of spinal manipulation. Stroke 2001;32:809-10, doi:10.1161/01.STR.32.3.809.

12. Caplan L, Chung CS, Wityk R, et al. New England Medical Center Posterior Circulation Stroke Registry: I. Methods, data base, distribution of brain lesions, stroke mechanisms, and outcomes. I Clin Neurol 2005;1:14-30, doi:10.3988/ jen.2005.1.1.14.

13. Kim J, Lee J, Suh D, et al. Spectrum of lateral medullary syndrome. Correlation between clinical findings and magnetic resonance imaging in 33 subjects. Stroke 1994;25:140510, doi:10.1161/01.STR.25.7.1405.

14. Tarnutzer AA, Berkowitz AL, Robinson KA, et al. Does my dizzy patient have a stroke? A systematic review of bedside diagnosis in acute vestibular syndrome. CMAf 2011;183: E571-91, doi:10.1503/cmaj.100174.

15. Kattah JC, Talkad AV, Wang DZ, et al. HINTS to diagnose stroke in the acute vestibular syndrome: three-step bedside oculomotor examination more sensitive than early MRI diffusion-weighted imaging. Stroke 2009;40:3504-10, doi:10. 1161/STROKEAHA.109.551234.

16. National Institute of Neurological Disorders and Stroke rtPA Stroke Sudy Group. Tissue plasminogen activator for acute ischemic stroke. N Engl 7 Med 1995;333:1581-7, doi:10.1056/NEJM199512143332401.

17. Hopyan JJ, Gladstone DJ, Mallia G, et al. Renal safety of CT angiography and perfusion imaging in the emergency evaluation of acute stroke. A7NR Am 7 Neuroradiol 2008;29: 1826-30, doi:10.3174/ajnr.A1257.

18. Hwang DY, Silva GS, Furie KL, et al. Comparative sensitivity of computed tomography vs. magnetic resonance imaging for detecting acute posterior fossa infarct. 7 Emerg Med 2012;42:559-65, doi:10.1016/j.jemermed.2011. 05.101.

19. Chalela JA, Kidwell CS, Nentwich LM, et al. Magnetic resonance imaging and computed tomography in emergency 
assessment of patients with suspected acute stroke: a prospective comparison. Lancet 2007;369:293-8, doi:10.1016/ $\underline{\text { S0140-6736(07)60151-2. }}$

20. Lindsay MP, Gubitz G, Bayley M, et al., Canadian best practice recommendations for stroke care (update 2010). On behalf of the Canadian Stroke Strategy Best Practices and Standards Writing Group. Ottawa: Canadian Stroke Network; 2010.

21. Engelter ST, Rutgers MP, Hatz F, et al. Intravenous thrombolysis in stroke attributable to cervical artery dissection. Stroke 2009;40:3772-6, doi:10.1161/STROKEAHA.109.555953.
22. CAST (Chinese Acute Stroke Trial) Collaborative Group. CAST: randomised placebo-controlled trial of early aspirin use in 20000 patients with acute ischaemic stroke. Lancet 1997;349:1641-9, doi:10.1016/S0140-6736(97)04010-5.

23. Nelles G, Contois KA, Valente SL, et al. Recovery following lateral medullary infarction. Neurology 1998;50:1418-22, doi:10.1212/WNL.50.5.1418.

24. Stewart P, Agur A, Leibgott B, et al. Functional neuroanatomy atlas. Toronto: The Functional Neuroanatomy Group, University of Toronto; 2003. 